\title{
Síndrome de Wilkie. Caso Clínico
}

\author{
Carolina Maya L. ${ }^{1}$, Felipe Zapata U. ${ }^{1}$, Jorge Vergara T. ${ }^{2}$ y Susana Cardona B. ${ }^{1}$
}

Departamento de Cirugía General Hospital General de Medellín.

2Departamento de Radiología Hospital General de Medellín. Medellín, Colombia.

Recibido 2020-11-17, aceptado 2021-05-08

Correspondencia a:

Dra. Carolina Maya L caromayalo@gmail.com

\section{Wilkie syndrome. Case Report}

Introduction: Wilkie syndrome is a rare cause of upper gastrointestinal tract obstruction, it is characterized by a decrease in the aorto-mesenteric angle that results in a compression of the third portion of the duodenum causing high obstructive symptoms. Case Report: We present the case of a 64-year-old woman, with a history of abdominal pain, heartburn, early satiety, and intolerance to the oral route, in whom initial studies showed esophagitis, but in whom symptoms did not improve with the classic treatment for peptic acid disease, additional imaging studies suggested Wilkie syndrome. Discussion and Conclusion: It is a pathology that must be known and suspected in patients with a history of weight loss, marked intolerance to oral intake, and lack of treatment response.

Key words: Wilkie syndrome; superior mesenteric artery syndrome; duodenal obstruction.

\section{Resumen}

Introducción: El síndrome de Wilkie es una causa poco frecuente de obstrucción del tracto digestivo superior, se caracteriza por una disminución del ángulo aorto-mesentérico que resulta en compresión de la tercera porción del duodeno que produce síntomas obstructivos altos. Caso Clínico: Presentamos el caso de una mujer de 64 años, con historia de dolor abdominal, pirosis, saciedad precoz e intolerancia a la vía oral, en quien los estudios iniciales mostraron esofagitis, pero en quien los síntomas no mejoraron con el tratamiento clásico para enfermedad ácido péptica y en quien estudios imagenológicos adicionales sugerían el síndrome de Wilkie como causa de los síntomas. Discusión y Conclusión: Se trata de una patología que debe conocerse y considerar en paciente con historia de pérdida de peso, marcada intolerancia a la vía oral y falta de respuesta al manejo.

Palabras clave: síndrome de Wilkie; síndrome de la arteria mesentérica superior: obstrucción duodenal.

\section{Introducción}

El síndrome de la pinza aorto-mesentérica, también conocido como síndrome de Wilkie o de la arteria mesentérica superior, es una forma poco frecuente de obstrucción de intestinal alta en la que el duodeno, a nivel de su tercera porción, se encuentra comprimido entre la arteria mesentérica superior y la aorta abdominal, dado por perdida del tejido graso presente a este nivel, la cual puede tener una etiología multicausal ${ }^{1}$. A pesar de ser descrita por primera vez hace más de 150 años, es hasta hoy poco conocida por lo que su diagnóstico continúa siendo un reto, haciendo que la mayoría de los pacientes tengan múltiples diagnósticos y tratamientos fallidos antes de lograr el tratamiento definitivo ${ }^{2}$.

\section{Caso clínico}

Mujer de 62 años, quien consulta en urgencia por cuadro de 2 años de evolución de dolor abdominal localizado en hipocondrio izquierdo, progresivo, asociado con saciedad precoz, pirosis, emesis posprandial con intolerancia marcada a alimentos sólidos y pérdida de peso aproximada de $10 \mathrm{~kg}$. Relata múltiples consultas a servicio de urgencias por este motivo en las que se da manejo sintomático, desde hace 1 mes en manejo ambulatorio por gastroenterología, con diagnóstico de enfermedad por reflujo gastroesofágico, en tratamiento con inhibidor de bomba de protones sin mejoría. En el momento cursando con crisis de dolor por lo cual consulta. Como antecedentes personales relevantes, refería enfermedad coronaria con necesidad de cirugía de 
revascularización miocárdica en 2014. Endoscopia digestiva superior extrainstitucional que reportaba esofagitis no erosiva y ecografía de abdomen total sin hallazgos patológicos. Al examen físico ingresa estable hemodinámicamente, con dolor a la palpación abdominal en hipocondrio izquierdo sin otros hallazgos patológicos. Se realiza tomografía contrastada de abdomen donde el hallazgo más relevante fue el reporte de dilatación de la cámara gástrica (Figura 1), primera y segunda porción del duodeno, asociado a disminución de la distancia aortomesentérica y del ángulo aortomesentérico, con una longitud de $3,7 \mathrm{~mm}$ (Figura 2) y $19^{\circ}$ (Figura 3) respectivamente.

Se realiza diagnóstico de síndrome de la arteria mesentérica superior por lo que se suspende vía oral y se inicia manejo con nutrición enteral, vía sonda nasoyeyunal, la cual tolera adecuadamente presentando además mejoría marcada del dolor abdominal. Al séptimo día del inicio de la nutrición enteral, se realiza radiografía de vías digestivas altas en la que se evidencia dilatación del estómago y del duodeno en la primera y segunda porción, con retraso del vaciamiento gástrico, sin identificar causa de la obstrucción. A los 12 días del inicio de la terapia nutricional, se decide iniciar vía oral con dieta líquida la cual tolera adecuadamente, se avanza hasta llegar a blanda sin reaparición del dolor ni emesis por lo que se da alta para continuar seguimiento ambulatorio.

\section{Discusión}

En 1861, Carl von Rokitansky, médico de la Universidad de Viena, en el tercer volumen de su obra Handbuch der patologischen Anatomie, describe por primera vez, lo que hoy conocemos como el síndrome de la arteria mesentérica superior o síndrome de Wilkie; se refiere a éste como una incarceración interna, en la que la porción transversa del duodeno es comprimida por el mesenterio del intestino delgado, específicamente a nivel de la arteria mesentérica superior, desde el frente hasta a la pared abdominal posterior rígida. En 1927 David Wilkie, describe 75 casos y en su honor se acuña el epónimo ${ }^{3}$.

Se han descrito múltiples factores de riesgo para el desarrollo de esta patología como es la presencia de estados catabólicos acelerados, desencadenado por enfermedades neoplásicas, quemaduras extensas, pacientes críticamente enfermos; así como trastornos alimenticios con pérdidas marcadas de peso, VIH, estados posoperatorios de cirugía bariátrica, corrección de escoliosis, el rápido aumento de la estatura sin aumento proporcional en el peso,

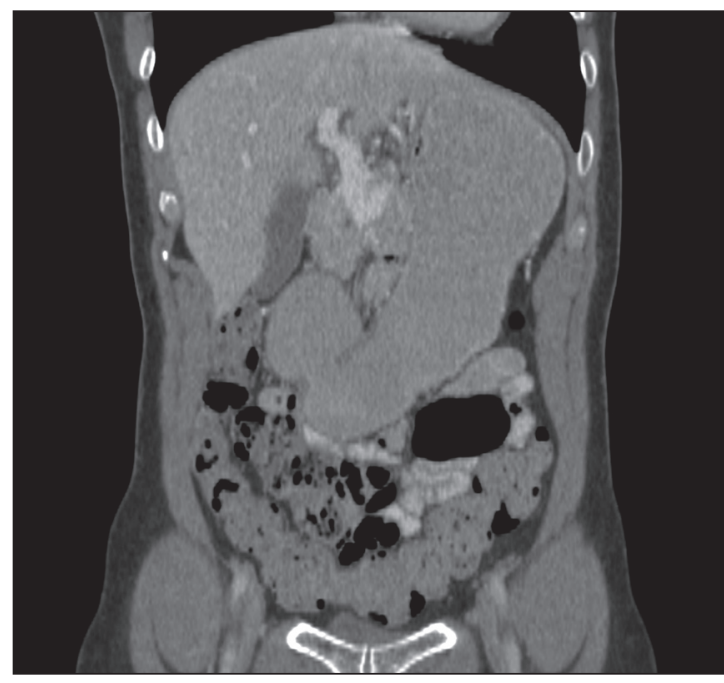

Figura 1. Corte coronal de una TC de abdomen y pelvis que evidencia dilatación gástrica.

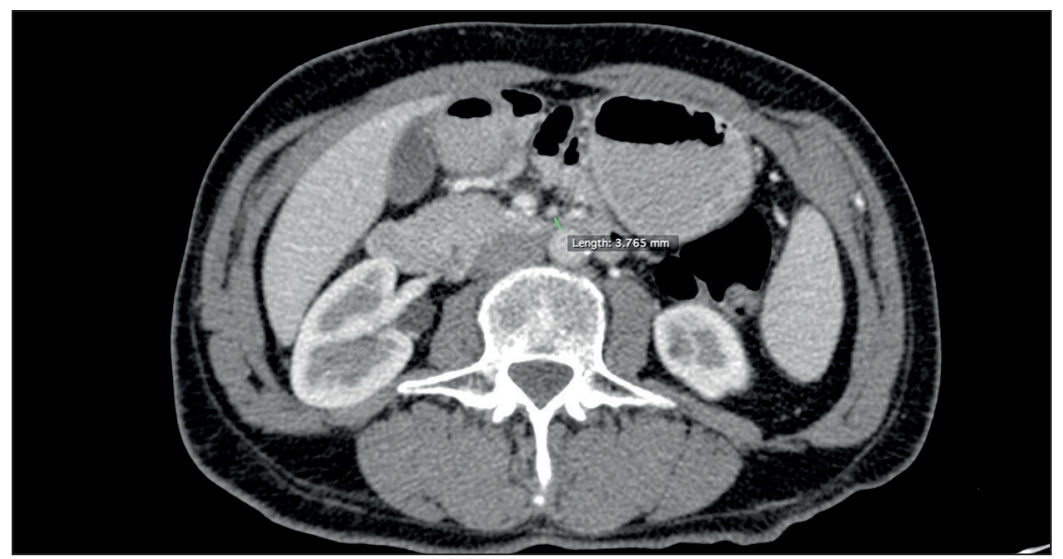

Figura 2. Corte axial de una TC de abdomen que evidencia distancia aortomesentérica de $3,7 \mathrm{~mm}$.
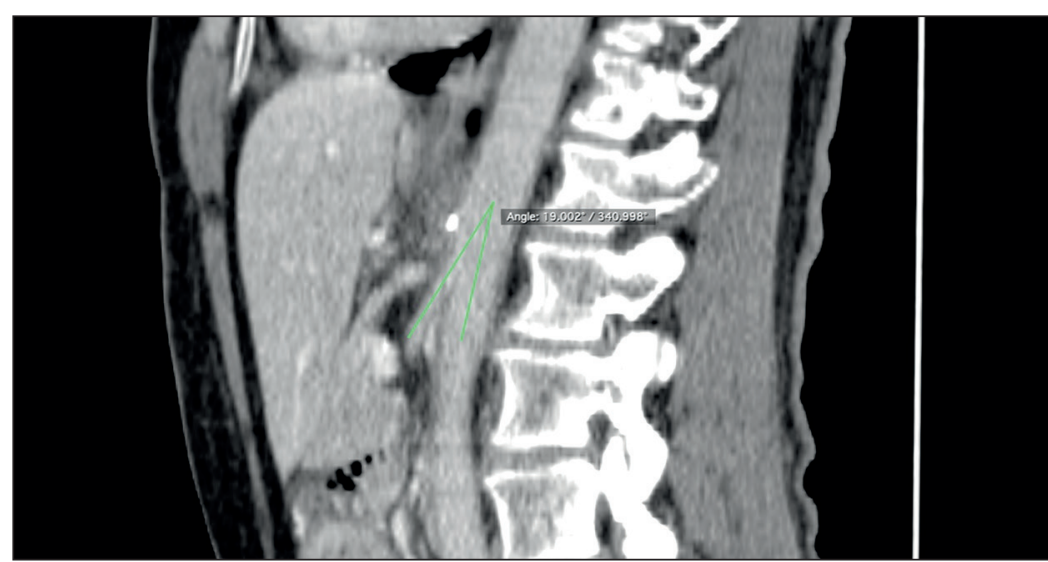

Figura 3. Corte sagital de una TC de abdomen que evidencia ángulo aortomesentérico de $19^{\circ}$ 
entre otros ${ }^{1,4-8}$ El resultado final es la disminución del cojinete graso mesentérico que produce un acortamiento del espacio entre la arteria mesentérica superior y la aorta, manifestado radiológicamente por una diminución del ángulo aortomesentérico cuyo valor normal varía entre $38-65^{\circ}$ y de $10-28 \mathrm{~mm}$ para la distancia aortomesentérica ${ }^{9}$; esto desencadena la compresión extrínseca de la tercera porción del duodeno a nivel de L1, nivel al cual el duodeno atraviesa la línea media.

La incidencia de esta patología es del 0,013$0,3 \%$ en la población general con incidencias mayores posterior a la corrección quirúrgica de escoliosis y en pacientes con quemaduras graves. La edad más frecuente de diagnóstico está entre 10 y 39 años, con una relación mujer-hombre de $3: 2^{9,10}$. Los pacientes cursan con un cuadro crónico caracterizado por dolor abdominal epigástrico postprandial, saciedad precoz, náuseas y emesis, reflujo gastroesofágico, eructos y anorexia secundaria por temor a desencadenar los síntomas; todo esto llevando a pérdida de peso y exacerbación de los síntomas que lo convierte en un ciclo vicioso ${ }^{9-12}$. Al no ser específicos, pueden presentarse en paciente con enfermedad por reflujo gastroesofágico, úlcera péptica y trastornos funcionales, por lo que el diagnóstico requiere de un adecuado estudio endoscópico e imagenológico ${ }^{13}$. La endoscopia digestiva superior como estudio inicial puede evidenciar compresión pulsátil a nivel de la tercera porción del duodeno ${ }^{3}$, en la radiografía de vías digestivas con bario se evidencia típicamente una dilatación de la primera y segunda porción del duodeno con o sin dilatación gástrica, sin embargo, es un hallazgo inespecífico. El estándar de oro para el diagnóstico en la actualidad es la tomografía computada contrastada, que además de evidenciar la dilatación duodenal, permite evaluar el ángulo aortomesentérico. Se toman como puntos de corte para el diagnóstico un ángulo aorto-mesentérico $<22^{\circ}$ con una sensibilidad del $42,8 \%$ y especificidad del $100 \%$, y una distancia aorto-mesentérica de $8 \mathrm{~mm}$ con sensibilidad y especificidad del $100 \%{ }^{10}$. En el caso presentado, la paciente cursaba con los síntomas típicos descritos y en cuanto a los hallazgos imagenológicos, se evidenció en la radiografía de vías digestivas la dilatación a nivel gástrico y de la primera y segunda porción del duodeno; la confirmación diagnóstica se realizó mediante tomografía contrastada al evidenciar un ángulo de $19^{\circ}$ y una distancia aortomesentérica de $6 \mathrm{~mm}$.

En primera instancia, el tratamiento médico tiene como objetivo recuperar el tejido graso del espacio aorto-mesentérico mediante nutrición hipercalórica por vía oral, en pequeñas porciones y con cambios en las posiciones al comer (prono o decúbito lateral izquierdo) si el paciente lo tolera; en casos de intolerancia total a la vía oral, mediante nutrición enteral o nutrición parenteral ${ }^{10}$, las tasas de éxito descritas para el manejo conservador son del $85 \%{ }^{2}$. Algunos pacientes con síntomas severos pueden requerir sonda nasogástrica para la descompresión gástrica asociado al uso de procinéticos ${ }^{9}$. En los casos en los que el manejo medico falla, la corrección quirúrgica está indicada; sin embargo, hasta el momento no está estandarizado el tiempo de terapia nutricional optimo antes de considerar la terapia fallida ${ }^{14}$ por lo que la decisión de operar debe ser individualizada.

En 1958, Strong propone un procedimiento que consiste en liberar el duodeno de todos sus puntos de anclaje, incluyendo del ligamento de Treitz ${ }^{15,16}$, una opción atractiva ya que evita la sección y posterior confección de anastomosis del tracto gastrointestinal, pero con tasas de recurrencia del $25 \%$ por lo que no es muy utilizada. Otras opciones descritas en la literatura son las gastroyeyunostomias, con alta tasa de persistencia de síntomas por lo que no es recomendada y la duodenoyeyunostomia ${ }^{14}$ con mejores tasas de éxito.

Como se mencionó previamente, el síndrome de Wilkie tiene una baja incidencia; sin embargo, es una patología que debe conocerse y considerar en paciente con síntomas dispépticos, en especial cuando están asociados a historia de pérdida de peso, marcada intolerancia a la vía oral y falta de respuesta al tratamiento. La baja frecuencia con la que se presenta hace que no existan hasta el momento guías de práctica clínica, pero entendiendo la fisiopatología de la enfermedad consideramos que la terapia nutricional es adecuada como primera medida, sin embargo, el tiempo indicado de tratamiento médico aún está por definirse y requerirá más estudios para definir el momento óptimo para llevar a tratamiento quirúrgico.

\section{Responsabilidades éticas}

Protección de personas y animales. Los autores declaran que para esta investigación no se han realizado experimentos en seres humanos ni en animales.

Confidencialidad de los datos. Los autores declaran que en este artículo no aparecen datos de pacientes.

Conflictos de interés: no hay. 


\section{Bibliografía}

1. González Hermosillo-Cornejo D, Díaz Girón-Gidi A, Vélez-Pérez FM, LemusRamírez RI, Andrade Martínez-Garza P. Síndrome de Wilkie. Reporte de un caso. Cir Cir. 2017;85:54-9.

2. Frongia G, Schenk J-P, Schaible A, Sauer P, Mehrabi A, Günther P. Food fear, quick satiety and vomiting in a 16 years old girl: It's bulimia, or maybe not...? A case report of Wilkie's syndrome (superior mesenteric artery syndrome). Int J Surg Case Rep. 2019;65:184-8.

3. Madhab G, Madsen J, Brems Dalgaard E, Bharadwaz A. Superior Mesenteric Artery Syndrome: A Rare Mimic of Common Causes of Upper Gastrointestinal Obstruction. Euroasian J HepatoGastroenterol. 2014;4:58-60.

4. Capitano S, Donatelli G, Boccoli G. Superior Mesenteric Artery SyndromeBelieve in it! Report of a Case. Case Rep Surg. 2012;2012:1-3.

5. Agarwal T, Rockall TA, Wright AR, Gould SWT. Superior mesenteric artery syndrome in a patient with HIV. JRSM 2003;96:350-1.

6. Ovalle-Chao C, Hinojosa-Martinez
LM, Gutierrez-Castillo A, Velazco-De La Garza JH, Flores-Villalba E, DíazElizondo JA, et al. Acute-onset of superior mesenteric artery syndrome following surgical correction of scoliosis: Case report and review of literature. J Pediatr Surg Case Rep. 2017;19:31-3.

7. Adson DE, Mitchell JE, Trenkner SW. The superior mesenteric artery syndrome and acute gastric dilatation in eating disorders: a report of two cases and a review of the literature. Int J Eat Disord. marzo de 1997;21:103-14.

8. Sun K-K, Wu X, Liu G, Qian H, Shen $\mathrm{X}$. Primary adenocarcinoma of the small intestine presenting as superior mesenteric artery syndrome: A case report. Oncol Lett. 2016;11:1903-6.

9. Mandarry MT, Zhao L, Zhang C, Wei $Z Q$. A comprehensive review of superior mesenteric artery syndrome. Eur Surg. 2010;42:229-36

10. Welsch T, Büchler MW, Kienle P. Recalling Superior Mesenteric Artery Syndrome. Dig Surg. 2007;24:14956.

11. Vásquez-Arango JN, Durán-Meléndez MA, Vásquez-Maya C. Tratamiento mínimamente invasivo del síndrome de
Wilkie. Rev Colomb Cir. 2018;33:299306

12. Requena-López AA, Mata-Samperio BK, Cuadra-Reyes LA, Casillas-Vargas R. Síndrome de Wilkie como etiología de oclusión intestinal en el adulto: a propósito de un caso. Cir Cir. 20 2020;88:2654

13. Baltazar U, Dunn J, Floresguerra C, Schmidt L, Browder W. Superior mesenteric artery syndrome: an uncommon cause of intestinal obstruction. South Med J. 2000;93:606-8.

14. Merrett ND, Wilson RB, Cosman P, Biankin AV. Superior Mesenteric Artery Syndrome: Diagnosis and Treatment Strategies. J Gastrointest Surg. 2009;13:287-92.

15. Matheus $\mathrm{C}$ de $\mathrm{O}$, Waisberg J, Zewer MH de T, Godoy AC de. Syndrome of duodenal compression by the superior mesenteric artery following restorative proctocolectomy: a case report and review of literature. Sao Paulo Med J. 2005;123:151-3.

16. Strong EK. Mechanics of Arteriomesenteric Duodenal Obstruction and Direct Surgical Attack Upon Etiology: Ann Surg. 1958;148:725-30. 